\title{
Changes in Coagulation and Fibrinolysis Systems During the Perioperative Period of Acute Type A Aortic Dissection
}

\author{
Chu-zhi Zhou, ${ }^{1,2}$ Yan-zhen Li, ${ }^{2}$ Ya-lan Yan, ${ }^{1}$ Dong-jie Feng, ${ }^{1}$ Min-xin Wei, ${ }^{2}$ Jun-min Wen ${ }^{1}$ \\ 'Department of Critical Care Medicine ICU, Fu Wai Hospital, Chinese Academy of Medical Sciences, Shenzhen, Shenzhen, \\ Guangdong, China; ${ }^{2}$ Graduate School of Tianjin Medical University, Tianjin, China
}

\section{ABSTRACT}

Background: Acute type A aortic dissection (ATAAD) has a high risk of perioperative bleeding and often requires extensive blood product infusions. Analysis of the changes in coagulation and fibrinolysis is both helpful for proper treatment and an improved prognosis. The present study investigated the changes in the coagulation and fibrinolysis systems during the perioperative period of ATAAD.

Methods: Twenty-two patients with ATAAD were included in this study. After diagnosis, all patients underwent ascending aorta replacement, aortic arch replacement, and implantation of a special stented endovascular graft. The control group included 25 patients undergoing elective aortic surgery. Baseline preoperative, intraoperative, and postoperative data were collected in both groups. Venous blood samples of all subjects were collected at five time points, after admission (T1), before surgery (T2), after protamine reversal (T3), postoperative $6 \mathrm{~h}$ (T4), and postoperative $24 \mathrm{~h}$ (T5), measuring the concentrations of platelet factor 4 (PF4), prothrombin fragment $1+2(\mathrm{~F} 1+2)$, tissue factor $(\mathrm{TF})$, tissue factor pathway inhibitor (TFPI), plasminogen activator (PA), plasminogen activator inhibitor-1 (PAI-1) and thrombin antithrombin complex (TAT) by enzyme-linked immunosorbent assays (ELISAs).

Results: The average age of the ATAAD group was $49.9 \pm 12.5$ years old, while that of the control group was $57.0 \pm 12.1$ years old. There were more patients with a smoking history, and the cardiopulmonary bypass time, aortic cross-clamp time, and preoperative left ventricular ejection fraction were higher in the ATAAD group than in the control group $(\mathrm{P}<0.05)$. Additionally, preoperative fibrin degradation products (FDP) and preoperative D-dimer were higher in the ATAAD group than in the control group $(P<0.05)$. However, time from onset to operation, intraoperative core temperature, preoperative B-type natriuretic peptide (BNP), and left ventricular end-diastolic diameter in the ATAAD

Received November 30, 2020; accepted fanuary 5, 2021

Correspondence: Min-xin Wei, Graduate School of Tianjin Medical University, Tianjin, 300070, China (e-mail: 239196788@qq.com); fun-min Wen, Department of Critical Care Medicine ICU, Fu Wai Hospital, Chinese Academy of Medical Sciences, Shenzhen, Shenzhen, Guangdong, China (e-mail: 1029013908@qq.com). group were lower than those in the control group $(P<0.05)$. In contrast, however, the proportion of abnormal bicuspid aortic valves in the control group was higher $(P<0.05)$. TF in the ATAAD group was significantly higher at T1 $(7.9 \pm 3.7$ $\mathrm{ng} / \mathrm{mL}$ versus $0.9 \pm 0.7 \mathrm{ng} / \mathrm{mL}, P<0.05)$. The TFPI in the ATAAD group was higher than that in the control group at T1 and T2 $(P<0.05)$. Additionally, PA in the ATAAD group was higher than that in the control group at T1, T2, T3, and T5 $(P<0.05)$, while PA in the control group was significantly higher at T3 than at T1 $(P<0.05)$. There was no significant difference in PAI-1 between the two groups before surgery $(P$ $>0.05)$. Nevertheless, both groups reached their peak value at T3. The platelet count and fibrinogen (FBG) in the ATAAD group decreased significantly from $\mathrm{T} 1$ to $\mathrm{T} 2$ and continued to decrease after cardiopulmonary bypass. F1+2 and TAT in the ATAAD group were higher than in the control group ( $P$ $<0.05)$; however, they peaked at T3. The PF4 in the ATAAD group slightly increased at T1, while PF4 at T3 was significantly higher than at $\mathrm{T} 1(P<0.05)$.

Conclusion: The changes in coagulation and fibrinolysis in the ATAAD group before surgery were very significant, which caused a large amount of fibrinogen and platelet consumption. Cardiopulmonary bypass (CPB) and a lower intraoperative core temperature exacerbated the coagulation and fibrinolysis disorder, and the pro-coagulant function of the platelets was activated after surgery. Maintaining the normal concentration of fibrinogen was helpful to correct the coagulation function disorder.

\section{INTRODUCTION}

Acute type A aortic dissection (ATAAD) is the most serious, urgent, and life-threatening type of aortic disease, and it often requires surgical treatment to avoid cardiac tamponade or sudden death. Bleeding is a common and severe complication during the perioperative period. After the blood flow enters the false lumen through the endometrial rupture, it leads to consumption coagulopathy [Geirsson 2018]. With the impairment of deep hypothermic circulatory arrest (DHCA), bleeding complications seriously affect the survival of patients. It has been reported that $20 \%$ of hospital mortality during the perioperative period of aortic dissection is related to coagulation [Zindovic 2015]. Postoperative bleeding, blood product transfusions, and surgical reoperations for persistent bleeding are the most prevalent and severe 
complications encountered [Paparella 2011]. A study by Guan et al. has identified activation of the coagulation system and consumption coagulopathy after ATAAD surgery, but their study lacks clinical applicability [Guan 2016]. We selected a group of patients undergoing elective ascending aorta or aortic root surgery for comparison, and at the same time, we tested and compared the changes before, during, and after surgery. The objective of the present study was to explore the changes in coagulation, fibrinolysis, and platelet activation during the perioperative period of ATAAD.

\section{MATERIALS AND METHODS}

Design and participants: This study was designed as a single-center prospective observational study comparing ATAAD patients with patients in a control group (referred to as the control group) who underwent ascending aorta or aortic root surgery under mild hypothermic cardiopulmonary bypass.

The inclusion criteria for the ATAAD patients included age $\geq 18$ years old, ethics committee approval (Institutional Review Board File 201710), signed the clinical research informed consent, a clinically confirmed diagnosis (acute type A aortic dissection) by contrast-enhanced computed tomography (CT) within 48 hours after its onset, and a surgical procedure performed under deep hypothermia circulatory arrest. The exclusion criteria for the ATAAD patients included patients with previous liver disease (congenital and acquired coagulopathy), those who had taken anticoagulant drugs and platelet inhibitors within seven days before the surgery, aortic intermural hematoma, and hybrid surgery patients.

The inclusion criteria for elective aortic surgery in the control group included age $\geq 18$ years old, ascending aortic aneurysm (undergoing ascending aortic replacement), or aortic root replacement.

Surgical management: A median incision was performed under general anesthesia with tracheal intubation. Selective cerebral perfusion was achieved via arterial cannulation of the right axillary artery. The cardiopulmonary bypass procedure started after the systemic heparinization (heparin dosage $300 \mathrm{U} / \mathrm{kg}$ and maintaining ACT at more than $480 \mathrm{~s}$ ). DHCA began when the nasopharyngeal temperature dropped to 20 to 25 degrees Celsius. Our institutional preference for these patients is to perform aortic arch replacement with a graft with four branches, and a particular stented endovascular graft is implanted in the true lumen of the descending thoracic aorta. When the artificial four-branch blood vessel is connected, a catheter can be intubated to restore the perfusion of the lower half of the body. Two branches of the artificial endograft are anastomosed with the left common carotid artery and the left subclavian artery. After the blood oxygen saturation rises above $85 \%$, rewarming is started, and the proximal quadrant artery is then anastomosed. After completing the graft anastomosis for the two major aortic vessels, the ascending aorta is then incised to complete the procedure by anastomosing the innominate artery with the fourth endograft branch vessel. The lowest intraoperative core temperature averaged $24.7 \pm 0.5^{\circ} \mathrm{C}$.

The procedures of the control group: A total of 15 cases of Bentall procedures, three cases of Wheat procedures, five cases of David procedures, and two cases of ascending aorta replacement procedures made up the control group. All procedures were performed under mild hypothermia $(30.9 \pm 1.2)$ and cardiopulmonary bypass.

The transfusion of allogeneic blood during the perioperative period in the study strictly adhered to the experts' consensus on blood management of patients undergoing cardiovascular surgery in our institution [Hu 2012]: When hemoglobin $(\mathrm{Hb})$ is $<80 \mathrm{~g} / \mathrm{L}, \mathrm{Hb}<60 \mathrm{~g} / \mathrm{L}$ during $\mathrm{CPB}$ or there is clear evidence of organ ischemia, red blood cells (RBCs) can be transfused. When the platelet count is $<50 \times 109 / \mathrm{L}$ and there is a risk of bleeding, platelets $(\mathrm{Plt})$ can be transfused. Fresh frozen plasma (FFP) can be infused when the prothrombin time (PT) is $>1.5$ times the normal value, or the absolute value is $>17 \mathrm{~s}$. FFP also can be infused when the international normalized ratio (INR) and the activated partial thromboplastin time (APTT) are $>1.6$ and $>2$ times the normal value, respectively, combined with bleeding. Fibrinogen can be transfused when there is bleeding, massive blood transfusions, or $\mathrm{FBG}<1 \mathrm{~g} / \mathrm{L}$.

Indicators and methods: We collected the patient baseline data, including gender, age, body mass index (BMI), history of hypertension, smoking history, time from onset to operation, preoperative $\mathrm{PaO} 2 / \mathrm{FiO} 2$, hyperlipidemia, a history of stroke, chronic kidney disease, fibrin degradation products (FDP), D-dimer (D-dimer), CPB time, aortic crossclamp time, DHCA time, postoperative mechanical ventilation time, and postoperative ICU stay time. At five different time points: After admission (T1), before surgery (T2), after protamine reversal (T3), postoperative $6 \mathrm{~h}(\mathrm{~T} 4)$, and postoperative $24 \mathrm{~h}$ (T5), we monitored the Plt count, FBG, thrombin antithrombin complex (TAT), prothrombin fragment $1+2$ $(\mathrm{F} 1+2)$, plasminogen activator $(\mathrm{PA})$, plasminogen activator inhibitor-1(PAI-1), platelet factor 4 (PF4), tissue factor (TF), and tissue factor pathway inhibitor (TFPI).

Blood collection: Venous blood samples of all subjects were collected with sodium citrate anticoagulation tubes at five time points: After admission (T1), before surgery (T2), after protamine reversal (T3), postoperative $6 \mathrm{~h}$ (T4), and postoperative $24 \mathrm{~h}$ (T5). The venous blood samples were centrifuged for 10 minutes at $3000 \mathrm{rpm}$. The supernatant was preserved at $-80^{\circ} \mathrm{C}$ until analysis, when we measured the concentrations of TAT, F1+2, PA, PAI-1, PF4, TF, and TFPI by enzyme-linked immunosorbent assays (ELISAs).

Statistical analysis: All statistical analyses were performed using IBM SPSS software version 23.0 (IBM Corporation, USA). All measurement variables were expressed as the mean \pm standard deviation, while a normal distribution test was performed. The comparison of measurement data conforming to a normal distribution between groups was performed by using a t-test. The rank-sum test was used for comparisons between groups that did not conform to the normal distribution. All count variables were described as the frequency and percentage, and comparisons between groups were carried out using the chi-square test. The measurement data from repeated measures were compared using repeated-measures analysis of variance. A $P$-value $<0.05$ was considered statistically significant. 


\section{RESULTS}

Baseline characteristics: From March 2018 to March 2020, 22 patients were enrolled in the ATAAD group, while 25 patients undergoing elective aortic surgery were assigned to the control group. The average age of the ATAAD group was $49.9 \pm 12.5$ years, while the BMI was $25.7 \pm 2.5 \mathrm{~kg} / \mathrm{m} 2$. The average age in the control group was $57.0 \pm 12.1$ years old, while the BMI was $23.8 \pm 2.9 \mathrm{~kg} / \mathrm{m} 2$. Notably, the time from onset to operation in the ATAAD group was short $(3.8 \pm 1.6$ days versus $8.5 \pm 12.3$ days, $P<0.05$ ).

The proportion of the ATAAD group with a smoking history, and the cardiopulmonary bypass time, aortic crossclamp time, and preoperative left ventricular ejection fraction were higher than in the control group $(P<0.05)$. Routine test results before surgery showed that the concentrations of FDP and D-dimer in the ATAAD group were significantly higher than those in the control group $(P<0.05)$. The time from onset to operation, intraoperative core temperature, preoperative $\mathrm{BNP}$, and left ventricular end-diastolic diameter in the ATAAD group were lower than those in the control group $(P<0.05)$. Nonetheless, the proportion of bicuspid aortic valve malformations in the control group was higher $(P<0.05)$. (Table 1$)$

Perioperative details: The ATAAD group experienced a longer CPB time $(187.8 \pm 25.2 \mathrm{~min})$, a longer aortic crossclamp time $(124.4 \pm 24.4 \mathrm{~min})$, and more intraoperative blood loss $(2.0 \pm 0.5 \mathrm{~L}$ versus $1.1 \pm 0.6 \mathrm{~L}, P<0.05)$, but the 24 -hour chest tube output was, however, similar to that of the control group $(445.2 \pm 211.1 \mathrm{ml}$ versus $408.8 \pm 196.8 \mathrm{ml}, P>0.05)$. The blood product transfusions (plasma, packed red blood cells, fibrinogen, platelets) in the ATAAD group was higher than that in the control group $(P<0.05)$. In the present study, there were no in-hospital deaths in the ATAAD group, and there was no statistically significant difference in postoperative complications (renal replacement therapy, stroke, and reoperation for bleeding) compared with the control group $(P>0.05)$. (Table 2)

Table 1. Baseline and surgical characteristics of the study population

\begin{tabular}{|c|c|c|c|}
\hline Age (years) & $49.9 \pm 12.5$ & $57.0 \pm 12.1$ & 0.516 \\
\hline BMI $(\mathrm{kg} / \mathrm{m} 2)$ & $25.7 \pm 2.5$ & $23.8 \pm 2.9$ & 0.429 \\
\hline History of hypertension, $\mathrm{n}(\%)$ & $15(68.2 \%)$ & $11(44.0 \%)$ & 0.100 \\
\hline Bicuspid aortic valve, $\mathrm{n}(\%)$ & $0(0 \%)$ & $5(20.0 \%)$ & 0.009 \\
\hline $\mathrm{DM}, \mathrm{n}(\%)$ & $1(4.5 \%)$ & $2(8.0 \%)$ & 0.541 \\
\hline Aortic regurgitation, $\mathrm{n}(\%)$ & $4(18.2 \%)$ & $7(28.0 \%)$ & 0.184 \\
\hline $\mathrm{PaO} 2 / \mathrm{FiO} 2(\mathrm{mmHg})$ & $232 \pm 107.5$ & $331.6 \pm 130.2$ & 0.074 \\
\hline Hyperlipidemia, n (\%) & $1(4.5 \%)$ & $2(8.0 \%)$ & 0.500 \\
\hline Onset to surgery (days) & $3.8 \pm 1.6$ & $8.5 \pm 12.3$ & 0.001 \\
\hline CPB time (min) & $187.8 \pm 25.2$ & $130.6 \pm 39.5$ & 0.036 \\
\hline Aortic cross-clamp time (min) & $124.4 \pm 24.4$ & $85.2 \pm 24.6$ & 0.016 \\
\hline DHCA time (min) & $20.4 \pm 4.3$ & 0 & 0.000 \\
\hline Lowest core temperature $\left({ }^{\circ} \mathrm{C}\right)$ & $24.7 \pm 0.5$ & $30.9 \pm 1.2$ & 0.001 \\
\hline LVEDD (mm) & $48.4 \pm 10.1$ & $55.6 \pm 12.2$ & 0.143 \\
\hline Preoperative LVEF (\%) & $60.3 \pm 1.7$ & $54.4 \pm 11.3$ & 0.000 \\
\hline Preoperative NT-proBNP & $515.3 \pm 110.3$ & $1290.1 \pm 797.5$ & 0.001 \\
\hline Preoperative D-dimer (mg/L) & $12.9 \pm 7.5$ & $0.6 \pm 0.4$ & 0.000 \\
\hline
\end{tabular}

BMI, body mass index; DM, diabetes mellitus; COPD, chronic obstructive pulmonary disease; CPB, cardiopulmonary bypass; DHCA, deep hypothermia circulatory arrest; LVEF, left ventricular ejection fraction; LVEDD, left ventricular end-diastolic diameter; NT-proBNP, N terminal pro B type natriuretic peptide 
Table 2. Postoperative data of the two groups of patients

\begin{tabular}{lccc}
\hline Variable & ATAAD group $(N=22)$ & Control group $(N=25)$ & $P$-value \\
\hline Intraoperative blood loss (L) & $2.0 \pm 0.5$ & $1.1 \pm 0.6$ & 0.001 \\
$24 \mathrm{~h}$ postoperative drainage $(\mathrm{ml})$ & $445.2 \pm 211.1$ & $408.8 \pm 196.8$ & 0.163 \\
FFP $(\mathrm{ml})$ & $304.5 \pm 345.3$ & $329.8 \pm 125.3$ & 0.665 \\
Platelet concentrate $(\mathrm{U})$ & $19.1 \pm 6.8$ & $5.0 \pm 3.1$ & 0.001 \\
Packed red blood cells (U) & $2.3 \pm 2.1$ & $1.1 \pm 0.5$ & 0.021 \\
Fibrinogen concentrate (g) & $1.8 \pm 1.2$ & $0.4 \pm 0.5$ & 0.001 \\
Mechanic ventilation time (hour) & $38.0 \pm 26.5$ & $22.6 \pm 29.3$ & 0.576 \\
Length of ICU (day) & $6.5 \pm 4.9$ & $5.3 \pm 2.7$ & 0.106 \\
Length of in-hospital (day) & $27.5 \pm 12.2$ & $26.1 \pm 12.3$ & 0.695 \\
RRT, $\mathrm{n}$ (\%) & $3(13.6 \%)$ & $1(4.0 \%)$ & 0.243 \\
Postoperative stroke, $\mathrm{n}(\%)$ & $2(9.1 \%)$ & $0(0 \%)$ & 0.127 \\
Reoperation for bleeding, $\mathrm{n}(\%)$ & $2(9.1 \%)$ & $0(0 \%)$ & 0.127 \\
In-hospital mortality, $\mathrm{n}(\%)$ & $0(0 \%)$ & $0(0 \%)$ & 1.000 \\
\hline
\end{tabular}

FFP, fresh frozen plasma; RRT, renal replacement therapy

Table 3. Changes of TF, TFPI, PA and PAI-1 during the perioperative period

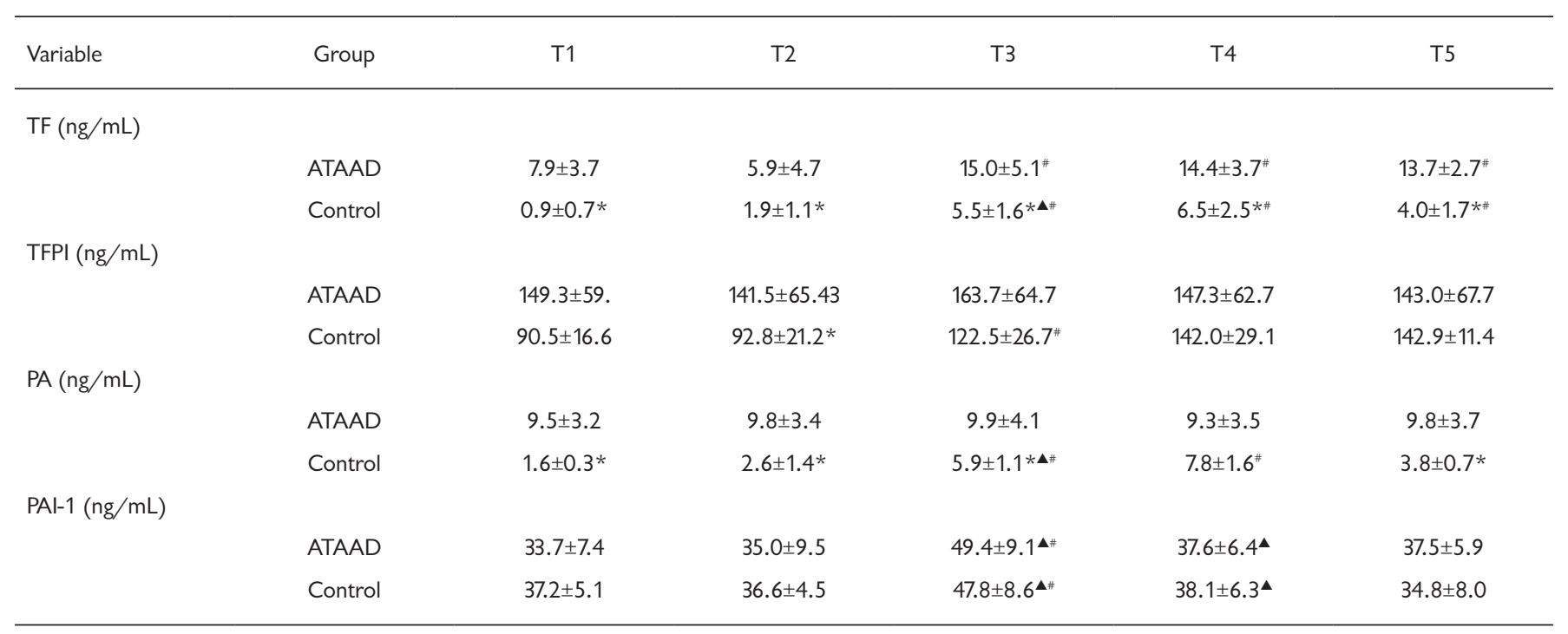

Comparison between the two groups, * indicates $\mathrm{P}<0.05$; comparison with previous time point intragroup, ${ }^{\mathbf{\Delta}}$ indicates $P<0.05$; Compared with the T1 intragroup, ${ }^{*}$ indicates $P<0.05$

Coagulation: Changes in the concentration of platelet counts, FBG, TAT, F1+2, PA, PAI-1, PF4, TF, and TFPI are shown in Table 3 and Figure 1. (Table 3 ) TF increased significantly at T1 in the ATAAD group $(7.9 \pm 3.7 \mathrm{ng} / \mathrm{mL})$, exceeding that in the control group $(0.9 \pm 0.7 \mathrm{ng} / \mathrm{mL})(P<0.05)$. At $\mathrm{T} 3, \mathrm{TF}$ in the two groups was significantly increased, and there was a significant difference compared with T1 in both groups, respectively $(P<0.05)$. TFPI was higher in the
ATAAD group at T1 and T2 than in the control group $(P<$ $0.05)$, and there was no significant concentration change in T2, while TFPI in the control group was significantly higher at T3 than T1 $(P<0.05)$. PA was higher in the ATAAD group at T1, T2, T3, and T5 than that in the control group $(P<$ $0.05)$, while the concentration in the control group was significantly higher at T3 than T1 $(P<0.05)$. There was no significant differences in PAI-1 between the two groups [T1 and 

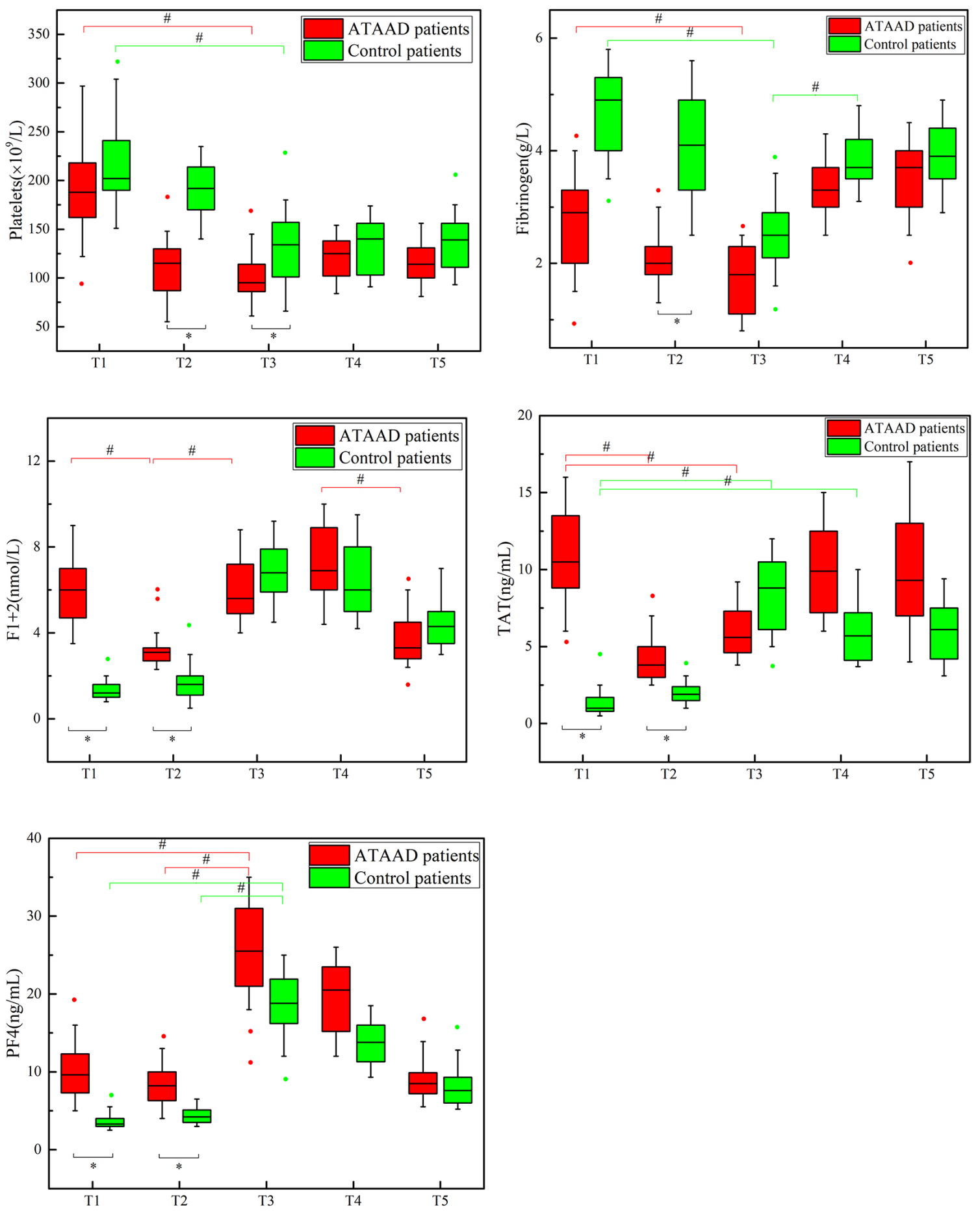

Figure 1. Box plots illustrating the levels of platelet, fibrinogen, TAT, F1+2, PF4; T1, after admission, T2, before surgery, T3, after protamine reversal, T4, 6 hours after surgery, and T5, 24 hours after surgery. NOTE: Comparison between groups, * showed $P<0.05$; Comparison intragroup, \# showed $P<0.05$. 
T2 $(P>0.05)]$, but the concentration in both groups reached a peak at $\mathrm{T} 3$.

In the ATAAD group, the Plt count and FBG decreased significantly from T1 to T2 and it continued to decrease after cardiopulmonary bypass. The concentration gradually increased after T4, although it rebounded slightly in both groups. The Plt count was still lower at T5 relative to T2. The concentration of F1+2 in the ATAAD group was significantly higher than that in the control group at T1 $(P<0.05)$. The concentration of $\mathrm{F} 1+2$ decreased gradually from $\mathrm{T} 1$ to T2 in both groups $(P<0.05)$, while it increased from T2 to T3 $(P<0.05)$. The change of TAT was similar to $\mathrm{F} 1+2$. PF4 in the ATAAD group increased slightly at T1, while it was significantly higher at T3 than T1 $(P<0.05)$. (Figure 1)

\section{DISCUSSION}

After the intimal tear of acute aortic dissection, the blood flow enters the false aortic lumen without endothelial coverage. The blood comes into contact with the subcutaneous collagen and the epigastric aortic membrane, and the tissue factors are released, presenting clinical manifestations of consumption coagulopathy [Cate 1975; Liu 2017]. This prospective observational study, using a control group of patients undergoing elective aortic surgery, confirmed that the coagulation and fibrinolysis systems were significantly activated before surgery, and cardiopulmonary bypass and hypothermia aggravated the disorders of coagulation and fibrinolysis.

Due to the exposure to collagen, subcutaneous tissue factor is released, and the aortic middle smooth muscle cells also secrete tissue factor. Then, the TF activates the system of coagulation, characterized by an increased concentration of TAT and F1+2 at T1, and the concentrations of D-dimer and FDP also were significantly higher than the normal range. These variables were all in the normal range in the control group.

We observed that after the destruction of the endothelial cells in the aortic intimal, a large amount of TF was released $(7.9 \pm 3.7 \mathrm{ng} / \mathrm{mL})$. Moreover, TF is the most effective catalyst for the exogenous coagulation cascade reaction, which would promote the formation of a thrombus in the false lumen [Gao 2019]. The concentrations of platelets and fibrinogen in the ATAAD group at T1 were slightly lower than those in the control group, but there was no significant difference between the two groups. However, the significant decrease in the concentrations of platelets and fibrinogen from $\mathrm{T} 1$ to $\mathrm{T} 2$ indicated that an increased consumption of clotting substrates $(\mathrm{P}<0.05)$ and the coagulation reaction in vivo continued under the trigger of TF. The changes of $\mathrm{F} 1+2$, TAT, $\mathrm{TF}$ and $\mathrm{PF} 4$ from $\mathrm{T} 1$ and $\mathrm{T} 2$, i.e., the concentrations of F1+2, TAT, TF, and PF4, decreased in the ATAAD group from onset to preoperation ( $3.8 \pm 1.6$ days). The resultant changes indicated that coagulation and platelet activation started the coagulation cascade at the onset of the disease; however, it consumed a large amount of coagulative factors.

Nonetheless, this effect gradually slowed down to a new steady state. The observed changes also are consistent with published reports in the literature [Suzuki 2009]. After surgery, the ATAAD patients underwent extracorporeal circulation, especially under the influence of DHCA, which showed an amplification effect on the production of thrombin and fibrinolytic enzymes. TF, TAT, F1+2, and PA were again increased at T3. We also observed that the concentration of TFPI and PAI-1 in the perioperative period (from T1 to T5) changed very little, and there was no significant difference between the two groups. The above-observed changes, however, showed that the anticoagulation and anti-fibrinolysis functions of the ATAAD patients were simultaneously activated. However, the concentrations of TFPI and PAI-1 were inhibited, and their coagulative reactions and fibrinolytic concentrations were not increased.

After the endometrial tears of the ATAAD patients, TF was released in large quantities and the coagulation cascade reaction was initiated, generating a large amount of thrombin with the release of $\mathrm{F} 1+2$ at the same time. Thrombin acts on FBG to produce fibrin, which further interacts with activated platelets to promote the formation of thrombosis in the false lumen [Stevic 2011]. To balance the hypercoagulability state, the plasminogen activator will activate plasminogen to produce plasmin, which then degrades fibrin and FBG into FDP and D-dimer, named secondary hyperfibrinolysis. In addition, the body also will combine its antithrombin with thrombin to produce TAT and TFPI to inhibit the exogenous coagulation pathway. In addition, to prevent excessive fibrinolysis, vascular endothelial cells synthesize and release PAI-1 to achieve the effect of inhibiting PA.

The ATAAD patients were in a contradictory state of systemic upregulated coagulation and fibrinolysis, which were inhibited by increased levels of TFPI and PAI-1. The coagulation and fibrinolysis systems were upregulated after onset in the ATAAD group; however, the increasing levels of TFPI and PAI-1 are not sufficient to completely compensate for the rapid increase in the concentration of thrombin and plasminogen activator, leading to upregulated levels of blood coagulation and fibrinolysis in ATAAD patients.

The activation of the coagulation system simultaneously activates platelet function, leading to platelet depletion. The platelet count decreased significantly in the ATAAD group before and during the operation, and the degree of thrombocytopenia has proven to affect the prognosis of ATAAD patients after surgery [Guan 2016]. In addition, the process of extracorporeal circulation also leads to platelet consumption and activation [Paparella 2006]. Hypothermia during ATAAD surgery affects platelet activation and aggregation, as well as reversible thrombocytopenia caused by the liver and spleen [Van Poucke 2016]. Thrombin production is synchronous with thrombocytopenia, which is the most prominent factor causing platelet activation in vivo [Edmunds 2006]. The present study highlighted a trend of decreased platelet count in patients with ATAAD before and during the operation, which was substantially significantly different from that in the control group. There was a significant difference between the ATAAD group and the control group at T3, indicating that the decreased platelet count was associated with extracorporeal circulation and the degree of hypothermia. Thrombocytopenia also increases the transfusion of platelets and packed red blood cells. In the present study, we measured the concentration of $\mathrm{PF} 4$, which reflects the degree of platelet activation. The results showed that the active function of platelets as 
reflected by PF4 increased after T3, which was also observed in the control group. These results showed that the coagulation ability of platelets was enhanced and not affected by the hypothermia. Previous research concluded that a higher concentration of PF4 may be related to the biological incompatibility, shear stress from the blood flow, heparin, and other reasons during CPB [Gupta 2009].

The concentration of fibrinogen in the ATAAD group had been significantly reduced before surgery, and the concentration of D-dimer was significantly higher than that of the control group before surgery. However, the concentration of fibrinogen at T3 in both groups was significantly lower than that at T1, especially in the control group, indicating many fibrinogen consumptions during surgery. A low concentration of fibrinogen at admission is considered an independent predictor of in-hospital mortality in patients undergoing ATAAD surgery [Liu 2018]. Notably, the hemostatic function and fibrinogen are markers of systemic reactions in the acute phase. After surgery, the concentration of fibrinogen was similar between the two groups, but the transfusion of fibrinogen in the ATAAD group was significantly higher than that in the control group. This suggests that there is still a continued fibrinogen consumption in ATAAD patients after surgery, possibly due to persistent thrombosis in the false lumen.

In summary, it was observed that the postoperative platelet count in the ATAAD group was significantly lower than that before surgery. However, if an appropriate concentration of fibrinogen is correctly maintained within the normal range, there will be no significant difference in the incidence of reoperations for bleeding and the volume of $24 \mathrm{~h}$ postoperative drainage compared with the control group. Fibrinogen plays a crucial role in the final formation of clots [Solomon 2011], and studies also have shown that increasing the fibrinogen concentration can compensate for the damaged platelet function [Velik-Salchner 2007]. The importance of an early fibrinogen infusion to correct coagulation disorders is also highlighted in the European guidelines [Schochl 2011]. Therefore, maintaining a normal fibrinogen range becomes an essential experience in correcting coagulation disorders in the ATAAD group after surgery.

The cardiopulmonary bypass process can cause blood dilution, a decreased platelet count, decreased coagulation factors, and a decreased fibrinogen concentration [Cate 1975], which also can be observed in the present study. Simultaneously, FDP and plasmin anti-plasmin complex (PAP) were both increased [Linden 2003].

Additionally, the influence of a low temperature can be seen between the two groups. The ATAAD group had a considerable influence of DHCA compared to the mild influence of the hypothermia observed in the control group. As shown in Table 3 and Figure 1, we observed an interesting phenomenon where $\mathrm{F} 1+2, \mathrm{PA}$, and TAT increased from $\mathrm{T} 2$ to $\mathrm{T} 3 \mathrm{in}$ the control group compared with the ATAAD group. A lower temperature would slow down the coagulation cascade reaction, decrease enzyme activities [Hardy 2004], and impair the function of the von Willebrand factor (vWF) [Van Poucke 2014]. Even if the clotting factor level is normal, lower temperatures can cause coagulation dysfunction.
Changes in the coagulation system in both groups were not completely reversed after $\mathrm{CPB}$. This further shows that $\mathrm{CPB}$ or hypothermia has a nonheparin-dependent deleterious effect on coagulation and fibrinolysis, which was more apparent in the ATAAD group compared with the control group. In the present study, intraoperative blood loss was significantly increased in patients undergoing ATAAD surgery, while postoperative blood loss and the postoperative incidence of reoperations for bleeding did not differ between the groups. Nonetheless, the differences may be attributed to the fact that ATAAD patients received more fibrinogen, platelets, and plasma infusions. Notably, patients undergoing anticoagulant and dual antiplatelet therapy were excluded from this study, which might have ruled out a population of patients at a considerably increased risk of bleeding.

Study limitations: In the present study, the data of the two groups were not identical at baseline, and they had different surgical parameters. There was no strictly standardized blood coagulation management. Additionally, the control group had no aortic dissection. In addition, the ATAAD patients had a lower intraoperative core temperature and a DHCA procedure, both of which would be expected to significantly affect the blood coagulation system. Since anticoagulant or antiplatelet therapy may be required 24 hours after surgery in both groups, to avoid interfering with the changes, we only observed the process of coagulation, fibrinolysis and platelet activation up to 24 hours after surgery.

\section{CONCLUSION}

The changes in the coagulation and fibrinolysis system in patients with ATAAD before surgery were significant, leading to the massive consumption of fibrinogen and platelets. Cardiopulmonary bypass and a lower intraoperative core temperature exacerbated the disorder of the coagulation and fibrinolysis, and the procoagulant function of platelets was activated after surgery. Maintaining a normal concentration of fibrinogen was helpful to correct the disorder of the coagulation function.

\section{REFERENCES}

Cate JW, Timmers H, Becker AE. 1975. Coagulopathy in ruptured or dissecting aortic aneurysms. Am J Med. 59:171-176.

Edmunds LH, Colman RW. 2006. Thrombin During Cardiopulmonary Bypass[J]. The Annals of Thoracic Surgery. 82(6): 2315-2322.

Gao Z, Pei X, He C, Wang Y, Lu J, Jin M, Cheng W. 2019. Oxygenation impairment in patients with acute aortic dissection is associated with disorders of coagulation and fibrinolysis: a prospective observational study. J Thorac Dis. 11(4):1190-1201.

Geirsson A, Ahlsson A, Franco-Cereceda A, et al. 2018. Hospital volumes and later year of operation correlates with better outcomes in acute type A aortic dissection. Eur J Cardiothorac Surg. 53:276-281.

Guan X, Li J, Gong M, et al. 2016. The hemostatic disturbance in patients with acute aortic dissection: A prospective observational study. Medicine (Baltimore). 95:e4710. 
Guan XL, Wang XL, Liu YY, et al. 2016. Changes in the hemostatic system of patients with acute aortic dissection undergoing aortic arch surgery. Ann Thorac Surg. 101:945-951.

Gupta SD, Pal S, Goswami A, et al. 2009. A Comparative Study to Determine the Effect of Intravenous Magnesium on Postoperative Bleeding after on Pump CABG in Patients Receiving Pre-Operative Aspirin.[J]. Indian Journal of Anaesthesia. 53(2):197-203.

Hardy JF, De Moerloose P, Samama M. 2004. Groupe d'intérêt en Hémostase Périopératoire. Massive transfusion and coagulopathy: pathophysiology and implications for clinical management. Can J Anaesth. 51:293-310.

Hu S, JI H, Sun H, et al. 2012. Consensus of experts on blood management of patients undergoing cardiovascular surgery [J]. Chinese Journal of Blood Transfusion. 31(4):321-325.

Linden MD. 2003. The hemostatic defect of cardiopulmonary bypass. J Thromb Thrombolysis. 16:129-47.

Liu Y, Han L, Li J, et al. 2017. Consumption coagulopathy in acute aortic dissection: principles of management[J]. Journal of Cardiothoracic Surgery. 12(1):51-58.

Liu J, Sun LL, Wang J, et al. 2018. The relationship between fifibrinogen and inhospital mortality in patients with type A acute aortic dissection. Am J Emerg Med. 36:741-744.

Paparella D, Galeone A, Venneri MT, et al. 2006. Activation of the coagulation system during coronary artery bypass grafting: comparison between on-pump and off-pump techniques. J Thorac Cardiovasc Surg. 131:290-297.
Paparella D, Rotunno C, Guida P, Malvindi PG, Scrascia G, De Palo M, et al. 2011. Hemostasis alterations in patients with acute aortic dissection. Ann Thorac Surg. 91:1364-1369.

Schochl H, Cotton B, Inaba K, et al. 2011. FIBTEM provides early prediction of massive transfusion in trauma. Crit Care. 15:R265.

Solomon C, Cadamuro J, Ziegler B, et al. 2011. A comparison of fifibrinogen measurement methods with fifibrin clot elasticity assessed by thromboelastometry, before and after administration of fifibrinogen concentrate in cardiac surgery patients. Transfusion. 51:1695-706.

Stevic I, Chan HH, Chan AK. 2011. Carotid artery dissections: thrombosis of the false lumen[J]. Thromb Res. 128(4): 317-324.

Suzuki T, Distante A, Zizza A, et al. 2009. Diagnosis of acute aortic dissection by d-dimer. The international registry of acute aortic dissection substudy on biomarkers (IRAD-Bio) experience. Circulation. 119:2702-2707.

Van Poucke S, Stevens K, Kicken C, et al. 2016. Platelet Function During Hypothermia in Experimental Mock Circulation[J]. Artificial Organs. 40(3):288-293.

Van Poucke S, Stevens K, Marcus AE, et al. 2014. Hypothermia: Effects on platelet function and hemostasis. Thrombosis J. 12:31.

Velik-Salchner C, Haas T, Innerhofer P, et al. 2007. The effect of fifibrinogen concentrate on thrombocytopenia. J Thromb Haemost. 5:1019-1025.

Zindovic I, Sjogren J, Bjursten H, et al. 2015. Impact of hemodynamic instability and organ malperfusion in elderly surgical patients treated for acute type A aortic dissection. J Card Surg. 30:822-9. 